\title{
Moral Reasoning Among Taiwanese Liberal Arts Students: Conventional Morality is not Enough
}

\author{
Jon Nichols \\ Department of Liberal Arts, Shih Chien University, Kaohsiung, Taiwan \\ Email address: \\ Tomballtxs66@yahoo.com (J. Nichols) \\ To cite this article: \\ Jon Nichols. Moral Reasoning Among Taiwanese Liberal Arts Students: Conventional Morality is not Enough. Education Journal. \\ Vol. 4, No. 5, 2015, pp. 207-213. doi: 10.11648/j.edu.20150405.14
}

\begin{abstract}
This study investigated the moral reasoning abilities of second-year university students in in a medium sized liberal arts university in Taiwan. General English students were asked to solve a moral dilemma and their responses were analyzed utilizing Kohlberg's theory of moral development. The results of the study indicate that the majority of student surveyed reason at the conventional level of morality, which is second stage of the second level on Kohlberg's three-level, sixstage rubric. The study goes on to question whether or not this level of moral reasoning meets the mandate of liberal arts education and whether or not this level of moral reasoning will be sufficient to equip students for the moral decisions which they will face in the future as leaders of policy and industry. Methods to increase students' moral reasoning ability are also dicussed within this study.
\end{abstract}

Keywords: Moral Reasoning, Taiwanese University Students, Kohlberg

\section{Introduction}

The idea of moral education has been in the forefront of Taiwanese educational discourse recently as evidenced by Chairman Tong's comments at the $31^{\text {st }}$ annual Academia Sinica meeting. In his opening remarks he pointed out the need for "virtuous" tertiary education in Taiwan and indicated that he thought many teachers were only pretending to teach and many students were only pretending to learn. He also extolled the Belgian education system where students focused on subjects such as history, art and culture, basic research, literature, physics and mathematics. He went on to say that Taiwan does not need more people who are only equipped to pass examinations, but who have no real skills or reasoning abilities [1].

This "examination culture" that exists in Taiwan also exists in South Korea, Japan, China and throughout East Asia Many of these students who have grown up within the "examination culture" have also received a limited amount of effective moral education, and as a result have become young adults who are incapable of higher level moral reasoning. This problem has recently been highlighted by a number of scandals involving international students from China such as the one that follows.

Admissions officials and others have reported finding falsified high-school transcripts, discrepancies between English-language test scores and a Chinese student's actual speaking ability, and phony letters of recommendation and essays. As many as 90 percent of recommendation letters for Chinese applicants to western universities were falsified in 2011, the most recent period studied, according to the U.S. educational consulting firm Zinch China. Seventy percent of admissions essays were written by someone other than the applicants, the firm found, and half of secondary school transcripts were doctored [2].

Unfortunately these types of scandals have become commonplace among Chinese international students. According to the WholeRen Education Company, (a U.S. company that works with Chinese students studying in American universities) dishonesty was a primary factor in more than $80 \%$ of expulsions of Chinese students from American universities [3]. This estimate was based on official data as well as a survey of more than 1600 Chinese students who were expelled from American universities. The survey was conducted amid reports from the American news media that federal prosecutors had indicted fifteen Chinese citizens for taking part in a fraud operation which was geared toward helping Chinese students gain admission into American universities [4]. According to Chen Hang, the chief development officer at WholeRen, "Chinese students used to be considered top-notch, but over the past five years their 
image has changed completely." [Now they are considered to be], "wealthy kids who cheat" [5]. Although some research has found significant differences between the moral reasoning of Mainland Chinese students and their Taiwanese counterparts in some regards, it stands to reason that Taiwanese students might succumb to the same pressures as their Chinese counterparts when faced with similar ethical dilemmas because of ethnic and cultural similarities [6]. Research also indicates that a willingness to cheat speaks to a lack of morality [7]. It is probable that this lack of morality will inform an individual's decisions not only as a university student, but also as a member of society for the remainder of his or her life.

The other side of the discourse is equally fascinating. How is it that some students are able to display advanced levels of moral reasoning? Why are some individuals willing to take moral stances which are both unexpected and come at a high personal cost? These questions are particularly salient when one considers the recent (spring 2014) political protests in Taiwan, where more than 100,000 Taiwanese citizens participated in what has become known as the Sunflower Student Movement. The protestors occupied both the Legislative and Executive houses (Yaun) of the Taiwanese government and demanded that a newly signed CrossStraight Trade Agreement with China be rescinded. These peaceful protests lasted for nearly a month before the protestors returned to their normal lives. As a result of the Sunflower Movement, the ruling KMT party suffered widespread defeats during the following elections [8].

Among the ways that the Sunflower Movement was unusual is the fact that it was led and organized by university students. Whereas student protests are the norm in many parts of the world, Taiwanese students are seen as being largely passive and compliant. It is interesting to many in the field of education what led these normally docile students to take up a political cause on such a massive scale and what type of moral thinking led them to participate. Regardless of whether or not educators agree with their students' methods or political perspective, it has to be acknowledged that these students acted on their own moral beliefs and were able to rise above the confines of filial, legal and societal conventions. This paper explores the moral development of Taiwanese university students and how the ESL classroom can be utilized as catalyst to encourage the development of higher-level moral reasoning.

\section{Related Literature}

\subsection{The Connection Between Critical Thought and Moral Development}

According to the world famous psychological theorist Jean Piaget, there are four stages of cognitive development. These stages are essentially biologically based and universal. Each person must complete the previous stage before entering into the subsequent stage and stages cannot be skipped. Piaget indicates that each individual progresses through the various stages at a different rate. By the time students reach university, Piaget's theory predicts that they should be well into the Formal Operation Stage. This is the final stage of cognitive development, and is characterized by the presence of critical thought and the ability to think about abstract concepts. The formal use of logic, systematic planning and deductive reasoning are also present within the thought processes of individuals who have reached the Formal Operational Stage. The ability to perform deductive reasoning is often a prerequisite for tasks involving science, higher level mathematics and literature. Deductive reasoning is an especially important feature of the Formal Operational Stage because it allows individuals to utilize general principles to determine a course of action within a specific situation. This has wide ranging applications for moral development and is one of the main areas where Piaget's theory of cognitive development and Kohlberg's theory of moral development converge. This relationship is an important one because a student's ability to reason at the highest moral levels is largely predicated upon their ability to engage in abstract thought [9].

\subsection{Lawrence Kohlberg Stages of Moral Development}

Lawrence Kohlberg was a developmental psychologist and Harvard professor who specialized in the area of moral development and moral education. He became famous in the 1970s for his research studies conducted at Harvard's Center for Moral Education [10]. His work on moral development grew out of Piaget's theories of cognitive development. Piaget had previously identified two stages of moral development within children, but Kohlberg was the first theorist to devote a significant body of research into developing a cohesive theory which explains moral reasoning. He subsequently expanded Piaget's two stage theory into a theory which encompasses six stages and three levels of development [11]. Kohlberg proposed that moral development is a process which continues through an individual's lifetime.

Kohlberg developed his theory based on interviews he conducted with students. Within these interviews the students were presented with moral dilemmas. The most famous scenario that Kohlberg used was called "The Heinz Dilemma". The following is a written transcript of the "Heinz Dilemma".

In Europe, a woman was near death from a special kind of cancer. There was one drug that the doctors thought might save her. It was a form of radium that a druggist in the same town had recently discovered. The drug was expensive to make, but the druggist was charging ten times what the drug cost him to make. He paid $\$ 200$ for the radium and charged $\$ 2,000$ for a small dose of the drug. The sick woman's husband, Heinz, went to everyone he knew to borrow the money, but he could only get together about $\$ 1,000$ which is half of what it cost. He told the druggist that his wife was dying and asked him to sell it cheaper or let him pay later. But the druggist said: 'No, I discovered the drug and I'm going to make money from it.' So Heinz got desperate and 
broke into the man's store to steal the drug-for his wife. Should the husband have done that? [12]

Kohlberg was interested not only in the answer that the students provided to the "Heinz" (and other) moral dilemma, but also the qualitative reasoning that went into how they arrived at their conclusions. After receiving the students' responses Kohlberg then classified them into one of three levels of moral development. These three levels were then subdivided into 6 stages - two stages per level. A brief explanation of these levels and stages follows.

\subsection{Preconventional Morality}

The first level of moral development is called Preconventional Morality. This type of moral reasoning is usually found in young children, but some adults also display a propensity to engage in this type of moral thought. As mentioned before, each level can be subdivided into two distinct stages. Kohlberg characterizes the first stage (The Obedience Stage) within the Preconventional level as moral reasoning which centers on concerns of obedience and punishment. During this stage, individuals see rules as static and absolute; they obey the rules in order to avoid punishment. In other words, a student who possesses this level of moral reasoning might approach the Heinz dilemma by stating that Heinz should not steal the medicine because he could be caught and put in prison. Heinz should avoid actions which could cause him to be punished. Conversely, a student might apply the same type of reasoning and arrive at the conclusion that Heinz should steal the medicine, because Heinz would feel really bad if his wife died and left him alone.

The second stage of development within the Preconventional Level is referred to as the Self-Interest Stage and features reasoning that is concerned with ideas of Individualism and Exchange. Here individuals are able to make moral decisions based on the needs of the individual. In other words, students at this level of moral development would advocate that Heinz take the course of action which best served his own purposes. Perhaps a student might answer that Heinz should steal the medicine because he will be happier if he saves his wife even if he is caught and punished. Students at this level of moral reasoning might also advocate that Heinz should refrain from stealing the medicine because if he were to be caught and sent to prison, the unpleasantness of incarceration would outweigh the joy of saving his wife.

\subsection{Conventional Morality}

The second level of moral reasoning is referred to as Conventional Morality. Moral decisions made by students within the first stage (The Conformity Stage) of this level are motivated by interpersonal relationships and fitting in with their peer group. Students within this stage are focused on following rules in order to be recognized as a good boy or good girl. There is an emphasis on conforming to social norms, and being nice is often considered more important that being morally correct. Individuals within this stage of moral reasoning can identify how moral choices can affect relationships. A student in this level might argue that Heinz should steal the medicine in order to fulfill the expectations within his marital relationship. A student might also indicate that Heinz should not steal the medicine because stealing is a criminal act and Heinz is not a criminal. Furthermore, since Heinz has already exhausted all of his legal options, he should bear no responsibility if his wife dies.

The second stage (The Law and Order Stage) of the Conventional Morality Level features reasoning that is centered on maintaining the social order. According to many researchers; this is the type of moral reasoning which is most commonly found in society in general [13]. Within this stage of moral thought, individuals consider society as a whole when making moral decisions. Individuals who reason at this stage focus on maintaining law and order by following rules. The importance of doing one's duty and respecting authority are also important considerations for individuals who reason at this level of moral development. A student who has attained this level of moral reasoning might advocate that Heinz should not steal the medicine because the law prohibits stealing, making it illegal. They might also reason that Heinz should take the medicine and accept the prescribed punishment - as well as paying restitution to the chemist. Individuals at this level reason that while it is important for Heinz to save his wife, it is equally important for social order to be maintained.

\subsection{Postconventional Morality}

Kohlberg's final level of moral reasoning is called the Postconventional level of Morality. Kohlberg felt that most people never reach this stage of moral reasoning even as adults. The first stage of Postconventional Morality is referred to as the Human Rights Stage. People who reason at this stage are able to see things from the point of view of people who possess different values and beliefs. Whereas individuals who have obtained stage 5 agree that laws are important for maintaining a well-functioning society, many stage 5 individuals also insist that these laws must be developed through a process which includes a consensus from the people who are expected to follow the laws. Students might solve the Heinz dilemma by stating that Heinz should steal the medicine because preserving life is more valuable than following the law. They might also argue that Heinz should not steal the medicine out of consideration for the chemist, who also has feelings and rights and deserves compensation for his discovery.

Kohlberg's final stage is the Universal Human Ethics stage. Individuals who have reached this stage of moral reasoning base their decisions upon universal ethical principles and abstract reasoning. At this stage, people are able to make moral judgements based on their own internalized principles of justice even when these principles conflict with existing laws [14]. Individuals who display stage six moral reasoning might tackle the Heinz dilemma by saying that Heinz should steal the medicine, because the preservation of human life is 
a fundamentally noble cause; and as such, should take precedence over preserving the property rights of another individual. They might also argue that Heinz should not steal the medicine because his actions could make the medicine more scarce which would in turn have a negative impact on other equally deserving patients [15].

\section{Methods}

\subsection{Research Design}

This study was designed as a case study with the purpose of ascertaining how students would respond to moral dilemmas within their natural environment. It is the goal of this paper to be able to make inductive theoretical generalizations and recommendations based on the students' responses to a moral dilemma. The procedure consisted of giving each of the students the "Heinz dilemma" and asking them to write an essay explaining what they thought Heinz should do. They were also asked to write a short essay on the following question: A week later Heinz is caught by the police and taken before the judge. What do you think Heinz's punishment should be? These questions were integrated into their English mid-term exams. The students gave their consent to be part of the research project at the beginning of the semester, but were not told until after their answers had been collected and collated that these specific questions were the questions which would be utilized for the purposes of this paper. The research questions were embedded into the students' written English exams in order to obtain information from a large-sized sample of subjects and also to insure that students would answer the questions honestly and would not skew the results by answering in a humorous or half-hearted manner.

\subsection{Participants}

The subjects comprised 285 sophomore students from various majors who attend a medium-sized private liberal arts university in southern Taiwan. The students were enrolled in either intermediate or upper-intermediate level English classes. The test was administered in written English. The average age of the students is 19.5 years old. Of the 285 participants, $194(68 \%)$ were female and 91(32\%) were male. Each of the students had taken various proficiency and placement tests in order to ensure that they were placed in either intermediate or upper-intermediate level English classes. The English classes were all required courses except for the most advanced class, which was an elective course.

\subsection{Data Analysis}

\section{Findings}

The students' responses were analyzed by an American licensed psychological counselor (L.P.C.), who categorized the responses into the appropriate three levels and six stages outlined in Kohlberg's theory of moral development. Of the 285 students who participated in the study, 164 (58\%) indicated that they thought that Heinz was right to steal the drug for his wife. The remaining 121 students (42\%) thought that Heinz was wrong to steal. Nearly all $(86 \%)$ of the students felt that Heinz deserved to be punished for his actions.

The majority (68\%) of the students' responses indicated that they should be placed in the second stage (L2S4) of The Conventional Level of Moral Development. Students in the second stage of the first level (L1S2) were the next biggest group with $13 \%$ of the students surveyed meeting the criteria for this category of moral reasoning. The next biggest group (11\%) was comprised of students who had attained the first stage of the third level of moral development. Students who had only attained the first stage of the first level (L1S1) and students in the first stage of the second level (L2S3) were equally distributed with.04\% each. There were no students who demonstrated that they had obtained the second stage of the third level of moral development.

\section{Discussion}

The vast majority of students tested, demonstrated that they reasoned at the second stage of the Conventional Level of morality. Most research suggests that the second stage of the conventional level of moral thinking is typically reached by students in late elementary school or early middle school. Many researchers suggest that even though this level of moral reasoning is typically reached by twelve-year olds, it is also a level of moral reasoning where many people stop progressing and as a result, this is the level and stage where most of society functions [16]. Whereas these results are not really surprising, educators within liberal arts universities must ask themselves if this level of moral reasoning is sufficient to meet the mandate of the liberal arts, and the challenges which will undoubtedly face Taiwan in the near future. Asked another way; should universities be training good citizens-people who merely follow the prescribed rules and regulations - or should they be striving to inspire the next generation of leaders? Is it enough to create a generation of young people who will follow all the rules and regulations of society, or should liberal arts programs strive to create an elite group of leaders who have the moral reasoning ability to shape the future course of policy and commerce throughout Taiwan? This is a question which seems to be on the minds of many of the educational leaders in Taiwan.

\subsection{Morality and Abstract Thinking}

At the heart of Kohlberg's theory of moral development is the idea that the qualitative progress through the different levels and stages of moral development is dependent on a concomitant development of cognitive abilities [17]. Piaget and Kohlberg both conceptualize the earlier stages of moral development as being characterized by decisions which are based on an immediate and concrete reward/punishment scheme; with the later stages being based on more abstract values which transcend ideas of personal loss and gain. In essence, the lower levels of moral reasoning are egocentric 
and depend on concrete cognitions while the higher levels rise above both conventional society and the individual, and seek to apply abstract universal principles to moral dilemmas. Kohlberg's levels of moral reasoning roughly correspond to the Concrete Operational and Formal Operational stages of cognitive development within Piaget's theory of cognitive development. Kohlberg's model is heavily dependent on the idea that moral reasoning is a product of cognitive development which is heavily influenced by experiences within the environment as well as social interactions. Kohlberg believed that in order for moral growth to occur, students must be presented with dilemmas which would facilitate reasoning at the next sequential higher stage of morality [18]. In this way, students would be able to develop the type of moral reasoning ability which would help them become leaders within society.

Kohlberg felt that teachers should engage their students in discussions which feature moral dilemmas wherein the teacher could demonstrate higher-level moral reasoning. Kohlberg felt that these discussions should take place within the confines of formal education and that most moral development took place through social interaction. The discussion approach is based on the insight that individuals develop as a result of cognitive conflicts which challenge the moral reasoning of their current stage [19]. In Taiwan, university students could be exposed to opportunities to engage in higher-level moral reasoning in a variety of scenarios within civics, history, and language classes. When taught well, each of these classes require students to engage in critical thinking, and each also involves the discussion of various ethical and moral issues. Language classes can be an especially effective vehicle for teaching critical thinking and moral reasoning because of the fact that language is used to communicate and formulate moral ideas which can subsequently be acted upon. Literature is also a rich source of ethical and moral dilemmas that teachers can use to engage their students in discussions which require critical thinking and model higher-level moral reasoning. Because of language's special connection to morality, and the fact that all university students in Taiwan are required to take English courses, it seems that providing students with the opportunity to develop higher-level moral reasoning skills within the context of ESL courses would be a widely accepted practice.

\subsection{Areas for Further Research}

\section{Utilizing a Discussion-based Approach to Moral Reasoning}

One of the main limitations of this study is the fact that the research question focuses only on ascertaining the current level of students' moral reasoning but does not implement any techniques for improving moral reasoning ability. As a result, Kohlberg's theory that moral reasoning could be enhanced by means of engaging students in discussions where moral dilemmas are presented and solved utilizing higher-level moral reasoning, has not been replicated within this study. It would be interesting to see of Kohlberg's theories hold true for a large sample of Taiwanese university students.

\subsection{Morality and Religion}

In follow-up discussions with test subjects the researcher asked students if there were any philosophical or religious concepts that they could apply to the Heinz dilemma. Students unanimously responded that they were not aware of how religion or philosophy could play any part in making a life and death decision such as the one that faced Heinz. This was an unexpected result, since many of the test subjects identify as themselves as having various religious affiliations. Some researchers link both level 2 stage 3 (L2S3) and level 3 stage 6 moral (L3S6) reasoning with religious ideology. They have identified religious dogma as being conducive to moral reasoning that centers on the blind adherence to rules and religious hierarchy (L2S3) while others point to the overarching themes of forgiveness, sacrifice, redemption and spiritual justice which are also integral parts to many religious belief systems [20]. Unsurprisingly, none of the test subjects achieved scores that would indicate that they were reasoning at either of these levels. It does bear further research to find out if students who attended religious institutions of higher education would achieve different results.

\subsection{Morality and the Sunflower Movement}

As mentioned above, the question of participation in student-led protest movements such as "The Sunflower Movement" is an interesting one. If one were to analyze the moral reasoning that led to these protests one would expect to find a core group of leaders who were able to reason at higher levels - specifically the first stage of level three-surrounded by an even larger group who reasoned at the first stage of level two. In other words the leaders of the movement probably saw the new cross-strait trade agreement as unfair and not representative of the wishes of the majority of Taiwan's constituency. Since individuals who reason at the first stage of the third level place great importance on the idea that laws must be developed through a process which considers the wishes and needs of the people who are expected to follow them, we can see that "The Sunflower Movement" probably grew out of this type of moral reasoning. However it is probably unrealistic to assume that the more than 100,000 people who participated in the protests all function at this high level of moral reasoning. It is more reasonable to assume that many of the participants had given little thought to the actual issues involved in the protest and instead joined in as a way to receive validation from their peer group, thus making the moral reasoning involved in their decision to participate in the protests more consistent with individuals who reason at the first stage of level two. It would be an interesting area for further research to actually interview the leaders of "The Sunflower Movement" as well as a representative sample of participants who were not part of the leadership group and compare their levels of moral reasoning.

\section{Conclusion}

The study shows that the vast majority of university students 
surveyed engage in highly conventional moral reasoning. Whereas these results are not unexpected, it does seem that part of the mandate for a liberal arts education is to inspire students to become the next generation of leaders. With this in mind it becomes apparent that enhancing students' higher-level moral reasoning abilities is a desirable goal for educators in Taiwan. This is because university students who are able to engage in higher-level moral reasoning are more likely to become the type of adults who will have a positive impact on society in the future. However, because of Taiwan's historical and cultural affinity for Confucian philosophy [21], many of the practices which Taiwanese universities have adopted are in direct opposition to this goal. Currently Taiwanese students are taught to follow rules and be good citizens and great emphasis is placed on familial and societal conformity. To compound matters, Taiwanese university students typically experience very little autonomy in regard to their education and very little guidance in non-conventional moral reasoning. The majority of students have little say about which university they will attend; they are allowed to choose between a few universities which their high-school exit exams allow them to be eligible to attend. Most students also do not have freedom to choose the subject they will major in. Students are not allowed to select the teachers they prefer, and are often forced to attend classes within the cohort of students that entered the university at the same time as they did. Taiwanese university students are also often required to sit in assigned seats, where they are referred to by student number instead of name. Each of these practices decreases the students' feelings individuality and emphasizes their membership in a larger group which in turn reinforces their tendency to engage in conventional moral decision making.

Whereas this system has many advantages in regard to maintaining order and providing adequate administrative services, this lack of student autonomy may also have unfortunate consequences in regard to students' ability to think critically and engage in higher-level moral reasoning. In fact, these types of practices are almost guaranteed to create students who lack independence and who are unable to rise above conventional moral reasoning. This unquestioning adherence to conventional morality may prove problematic when the current generation of students becomes political and industry leaders faced with problems which their current level of moral reasoning ability is inadequate to handle. As educators we must ask ourselves if conventional morality will be enough to meet the challenges that our students face, or should we be striving to train our students to shape the course of society utilizing higher-level moral reasoning and universal principles.

\section{References}

[1] Yang C-Y. When a bunch of Taiwanese people pretend to teach and study it doesn't help the Country at all. The News Lens [Internet]. 2014Feb; Retrieved from: http://www.thenewslens.com/post/52164/
[2] Pratt, T. (2014, July 1). Concerns rise about cheating by Chinese applicants to U.S. colleges. The Hechingerreport. Retrieved from http://hechingerreport.org/concerns-risecheating-chinese-applicants-u-s-colleges/

[3] Qi, L. (2014, May 25). U.S. Schools Expelled 8,000 Chinese Students. The Wall Street Journal. Retrieved from Qi http://blogs.wsj.com/chinarealtime/2015/05/29/u-s-schoolsexpelled-8000-chinese-students-for-poor-gradescheating/?mod=e 2 twy

[4] "8 Plead Not Guilty in Chinese Test-Taking Scheme for Visas." The New York Times 17 June 2015. Web. $<$ http://www.nytimes.com/aponline/2015/06/17/us/ap-uschinese-testing-indictment.html?_r=1>.

[5] Qi, L. (2014, May 25). U.S. Schools Expelled 8,000 Chinese Students. The Wall Street Journal. Retrieved from Qi http://blogs.wsj.com/chinarealtime/2015/05/29/u-s-schoolsexpelled-8000-chinese-students-for-poor-gradescheating/?mod=e2twy

[6] Yuana, B., \& Shenb, J. (1998). "Moral Values Held by Early Adolescents in Taiwan and Mainland China." Journal of Moral Education, 27(2).

[7] Semerci, Ç. (2006). The Opinions of Medicine Faculty Students regarding Cheating in Relation to Kohlberg's Moral Development Concept. Social Behavior and Personality, 34(41).

[8] Harrison, M. (2014, April 1). The Sunflower Movement in Taiwan. Retrieved from http://www.thechinastory.org/2014/04/the-sunflowermovement-in-taiwan/

[9] Kohlberg, L., Levine, C., \& Hewer, A. (1983). Moral stages: A current formulation and a response to critics. Basel, New York: Karger.

[10] Barger, Robert N. Ph.D. "A Summary of Lawrence Kohlberg's Stages of Moral Development." A Summary of Lawrence Kohlberg's Stages of Moral Development. University of Notre Dame Notre Dame, 2000. Web. $<$ http://www.csudh.edu/dearhabermas/kohlberg01bk.htm>.

[11] Piaget, J. (1932). The Moral Judgment of the Child. London: Kegan Paul, Trench, Trubner and Co..

[12] Kohlberg, L. (1981). Essays on Moral Development, Vol. I: The Philosophy of Moral Development. San Francisco, CA: Harper \& Row.

[13] Colby, A; Kohlberg, L. (1987). The Measurement of Moral Judgment Vol. 2: Standard Issue Scoring Manual. Cambridge University Press.

[14] Kohlberg, L. (1973). "The Claim to Moral Adequacy of a Highest Stage of Moral Judgment". Journal of Philosophy (The Journal of Philosophy, Vol. 70, No. 18) 70 (18): 630-646. doi:10.2307/2025030. JSTOR 2025030.

[15] Kohlberg, L. (1981). Essays on Moral Development, Vol. I: The Philosophy of Moral Development. San Francisco, CA: Harper \& Row.

[16] Kohlberg, L; T. Lickona, ed. (1976). "Moral stages and moralization: The cognitive-developmental approach". Moral Development and Behavior: Theory, Research and Social Issues. Holt, NY: Rinehart and Winston. 
[17] Lee, Lee C. "The Concomitant Development of Cognitive and Moral Modes of Thought: A Test of Selected Deductions from Piaget's Theory." Genetic Psychology Monographs 83.1 (1971).

[18] Power, F., Kohlberg, L., \& Higgins, A. (1991). Lawrence Kohlberg's Approach to Moral Education (Critical Assessments of Contemporary Psychology). New York, New York: Columbia University Press.

[19] Power, F., Kohlberg, L., \& Higgins, A. (1991). Lawrence Kohlberg's Approach to Moral Education (Critical Assessments of Contemporary Psychology). New York, New York: Columbia University Press.
[20] Gibson, T. (2004). Proposed Levels of Christian Spiritual Maturity. Journal of Psychology and Theology, 32(4).

[21] Yuana, B., \& Shenb, J. (1998). "Moral Values Held by Early Adolescents in Taiwan and Mainland China." Journal of Moral Education, 27(2). 